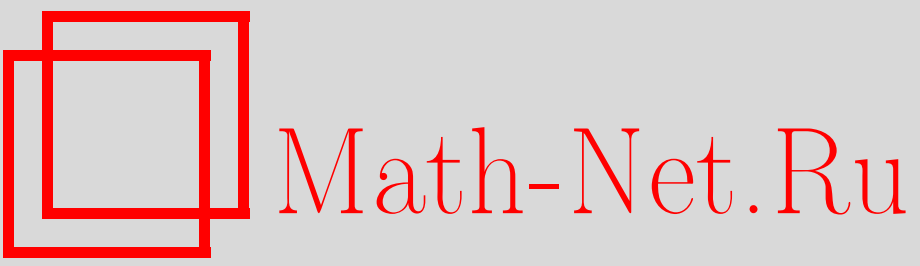

Д. В. Ширков, Ренормгруппа, причинность и нестепенная теория возмущений в КТП, ТМФ, 1999, том 119, номер 1, 55-66

DOI: https://doi.org/10.4213/tmf727

Использование Общероссийского математического портала Math-Net.Ru подразумевает, что вы прочитали и согласны с пользовательским соглашением

http://www . mathnet.ru/rus/agreement

Параметры загрузки:

IP : 3.85 .5 .30

26 апреля 2023 г., $18: 33: 45$ 
ТЕОРЕТИЧЕСКАЯ

И МАТЕМАТИЧЕСКАЯ

ФИЗИКА

Том 119, № 1

апрель, 1999

(C) 1999 г.

Д.В. Ширков*

\section{РЕНОРМГРУППА, ПРИЧИННОСТЬ И НЕСТЕПЕННАЯ ТЕОРИЯ ВОЗМУШЕНИЙ В КТП}

Структура квантово-полевого разложения наблюдаемых изучается в рамках новой "причинной аналитической версии" теории возмущений в квантовой хромодинамике. Здесь инвариантный заряд КХД $a\left(Q^{2} / \Lambda^{2}\right)=\beta_{1} \alpha_{s}\left(Q^{2}\right) / 4 \pi$ преврашается в $Q^{2}$-аналитическую инвариантную функцию $a_{\text {ан }}\left(Q^{2} / \Lambda^{2}\right) \equiv \mathcal{A}(x)$, которая по построению свободна от "призрачных" сингулярностей за счет появления непертурбативных вкладов.

В рамках аналитизированной теории возмушений разложение для наблюдаемой $F$ вместо степеней аналитического инвариантного заряда $\mathcal{A}^{n}(x)$ содержит специфические функции $\mathcal{A}_{n}(x)=\left[a^{n}(x)\right]_{\text {ан }}-n$-е степени $a(x)$, аналитизированные как целое. Анализ показал, что функции $A_{n>2}(x)$ в области малых $Q^{2} \leqslant \Lambda^{2}$ оказываются осциллирующими, что и приводит к слабой петлевой и схемной зависимостям. Таким образом, степенное разложение теории возмущений для $F(x)$ в результате процедуры аналитизации переходит в асимптотическое разложсение типа Эрдейи по нестепенной последовательности $\left\{A_{n}(x)\right\}$. Рассмотрены вопросы неоднозначности механизма причинной аналитизации и возможной несовместности некоторых ее версий со структурой ренормгруппы.

\section{1. ВВЕДЕНИЕ}

В работах $[1,2]$ механизм инвариантной аналитизации был применен к пертурбативной квантовой хромодинамике (пКХД). Он позволяет осуществить совмешение двух идей: ренормгруппового суммирования “больших" ультрафиолетовых (УФ) логарифмов и свойства аналитичности по переменной $Q^{2}$, отвечающего спектральному представлению $^{1)}$ типа Челлена-Лемана $[3,4]$ (см. ниже формулу (1)). Эта комбинация была впервые реализована в КЭД около сорока лет назад [6] для устранения призрачного полюса Фрадкина-Ландау.

\footnotetext{
1)Это представление является воплошением [5, гл. “Дисперсионные соотношения"] общих свойств локальной КТП, среди которых важную роль играет условие причинности. В данном контексте наряду с термином "аналитичность" мы будем иногда использовать в качестве синонимов "спектральность" и "причинность".
}

* Лаборатория теоретической физики им. Н. Н. Боголюбова, ОИЯИ, Дубна, Россия. E-mail: shirkovd@thsun1.jinr.ru 
Центральным объектом ренорминвариантной квантово-полевой теории возмушений является особая функция, так называемый инвариантный заряд или "инвариантная константа связи" $\alpha\left(Q^{2} / \Lambda^{2}\right)$, через которую выражаются наблюдаемые величины. Ввиду некоторой двусмысленности последнего выражения мы предпочитаем говорить об “инвариантной (функции) связи". В КХД параметр $\Lambda$, отвечающий положению нефизической особенности (полюса или начала ветвления), примерно равен 300 МэВ. В результате аналитизации подходящим образом нормированная инвариантная связь $a(x)=$ $\beta_{1} \alpha_{s}(x) / 4 \pi\left(\beta_{1}=11-2 n_{f} / 3\right)$ преврашается в новую аналитическую функцию связи $a_{\text {ан }}(x) \equiv \mathcal{A}(x)$, свободную от призрачных сингулярностей.

По построению аналитическая функиия связи определена представлением Челлена-Лемана

$$
\mathcal{A}(x)=\frac{1}{\pi} \int_{0}^{\infty} \frac{\rho(\sigma) d \sigma}{\sigma+x}
$$

где спектральная плотность $\rho(\sigma)=\Im a(-\sigma)$ вычислена на основе "исходного", просуммированного по ренормгруппе выражения $a(x)$.

В общем случае $\mathcal{A}(x)$ отличается от $a(x)$ на непертурбативные слагаемые ${ }^{2)}$, которые "вычитают" нефизические сингулярности, такие как призрачньй полюс (см. ниже формулы (2) и (6)).

Детальный анализ показал $[1,2]$, что аналитическая функция связи $\mathcal{A}(x)$ имеет несколько важных свойств. Она оказывается поразительно стабильной в инфракрасной (ИК) области (при $Q<\Lambda$ ) по отношению к многопетлевым поправкам и к выбору схемы перенормировки. Ее ИК-предельное значение $\mathcal{A}(0)$ в этом смысле является универсальным. Мы кратко обозреваем этот сюжет в п. 2.1-2.3.

С другой стороны, причинная аналитизация физических амплитуд не является однозначной операцией. Возможны несколько сценариев. В работе [8] была предложена и разработана особая версия - аналитическая теория возмущений (АТВ). Из-за специфического рецепта аналитизации вместо разложения по степеням теории возмушений, привычного для теоретической физики и КТП, аналитизированная физическая амплитуда $\mathcal{F}(x)$ оказывается представленной здесь в виде разложения более обшей формы - разложения по асимптотической последовательности функций $\mathcal{A}_{n}(x)=\left[a^{n}(x)\right]_{\mathrm{aн}}-$ " $n$-х степеней $a(x)$, аналитизированных как целое". В версии АТВ наблюдалось сильное уменьшение зависимости от вкладов высших петель и от схемы перенормировки (см. [8-10]).

Чтобы понять природу такого "петлевого и схемного иммунитета", в разделе 3 мы изучаем свойства нестепенной последовательности $\left\{\mathcal{A}_{n}(x)\right\}$, возникающей при использовании рецепта аналитизации АТВ.

В разделе 4 рассмотрена структура возможных вариантов аналитизации наблюдаемой и обсуждается опасность противоречия, точнее несовместности с внутренней структурой ренормгруппы, для некоторых из этих вариантов.

\footnotetext{
2) По поводу связи между ренорминвариантностью, причинностью и неаналитичностью по $\alpha$ см. также наши работы [7].
} 


\section{2. РЕШЕНИЕ РГ-УРАВНЕНИЙ И АНАЛИТИЗАЦИЯ}

2.1. Однопетлевая аналитизация $a(x)$. В однопетлевом приближении инвариантная функция связи $a(x)=1 / \ln x$ имеет полюс при $x=1$, несовместный со спектральным представлением (1). Аналитизация состоит в аналитическом продолжении выражения $1 / \ln x$ в область отрицательных $x$ и определении спектральной плотности $\rho(\sigma)=\pi\left(\ln ^{2} \sigma+\pi^{2}\right)^{-1}$ через его мнимую часть. Результирующий спектральньй интеграл может быть вычислен явно:

$$
a(x)=\frac{1}{\ln x} \Rightarrow a_{\mathrm{aH}}(x)=\mathcal{A}(x)=\frac{1}{\ln x}-\frac{1}{x-1} .
$$

Второй член, в точности компенсирующий призрачный полюс, имеет непертурбативную природу. При малых $a_{\mu}=a\left(Q^{2}=\mu^{2}\right)$ он не "оставляет следов" при разложении в ряд Тейлора, будучи пропорциональным $\exp \left(-1 / a_{\mu}\right)$. Для того чтобы увидеть это, следует от $\Lambda$-параметризации вернуться к параметризации через перенормированную константу связи $a_{\mu}$ и квадрат импульса отсчета $\mu^{2}$

Заметим, что связь между $\Lambda / \mu$ и $a_{\mu}$ в результате аналитизации меняется. Вместо обычного выражения $\Lambda^{2}=\mu^{2} \exp \left(-1 / a_{\mu}\right)$ мы приходим согласно (2) к неявному соотношению

$$
\Lambda^{2}=\frac{\mu^{2}}{f(a)} ; \quad a=\frac{1}{\ln f(a)}+\frac{1}{1-f(a)} .
$$

Здесь при малых $a$ (так же как и в (2) при больших $x$ ) можно пренебречь вторым непертурбативным членом. Однако при $a \sim 1(x \leqslant 1)$ этот член становится основным и приводит к фиксированной ИК-точке при $a=1$.

Аналитическая инвариантная связь $\mathcal{A}(x)$ является монотонной функцией на интервале $(0, \infty)$ и имеет конечный ИК-предел. Второй член в (2) быстро убывает при $x \rightarrow \infty$ : он составляет около $5 \%$ при $Q=10 \Lambda$ и всего $1 \%$ при $Q=25 \Lambda$. Полный набор решений (2), рассматриваемых как функции $Q^{2}$ с различными значениями параметра $\Lambda$, образует пучок с обшей точкой $\mathcal{A}(0)=1$. Это значение, соответствующее $\alpha_{s}(0)=4 \pi / \beta_{1}$, оказывается универсальным (см. ниже п. 2.3).

2.2. Двух- и трехпетлевой случаи. В двухпетлевом случае инвариантная связь определяется из неявного соотношения

$$
\frac{1}{a^{(2)}(x)}-b \ln \left(1+\frac{1}{b a^{(2)}(x)}\right)=\ln x ; \quad b=\frac{\beta_{2}}{\beta_{1}^{2}}\left(=\frac{64}{81} \text { при } n_{f}=3\right),
$$

получаемого интегрированием двухпетлевого дифференциального уравнения.

Итеративная процедура разрешения этого соотношения дает приближенное решение в явном виде

$$
a_{\text {итер }}^{(2)}(x)=\frac{1}{\ell+b \ln (1+\ell / b)}, \quad \ell=\ln \frac{Q^{2}}{\Lambda^{2}}=\ln x
$$

которое использовалось в наших предыдущих работах. 
Точное решение уравнения (3) может быть также выражено $[11,12]$ через специальную функцию $W$ - функцию Ламберта $W(z) e^{W(z)}=z$ (в обозначениях работы [13]), обладающую бесконечным числом ветвей $W_{n}(z)$ :

$$
a^{(2)}(x)=-\frac{1}{b} \frac{1}{1+W(z)} ; \quad z=-\frac{1}{e} x^{-1 / b}=-e^{-\ln x / b-1} .
$$

Бо́льшая часть физической области $x>1(z<-1 / e)$ соответствует ветви $W_{-1}(z)$, которая действительна и монотонна при $x \in(1, \infty)$. Отношение между $z$ и $x$ в $(5)$ определено из соображения соответствия с приближенным решением (4) при $x \gg 1$. Эта ветвь $\mathcal{W}(x) \equiv W_{-1}(z)$ комплексна ниже призрачной особенности при $x=1, z=-1 / e$.

Качественный анализ уравнения (3) или его точного решения обнаруживает, что эта особенность имеет форму корневой точки ветвления

$$
a^{(2)}(x \simeq 1)=\frac{1}{\sqrt{2 b(x-1)}}-\frac{1}{3 b}+O(\sqrt{x-1}) \quad(x=1+|\varepsilon|),
$$

которая приводит к нефизическому разрезу $0 \leqslant x \leqslant 1$.

Таким образом, отправляясь от двухпетлевого решения (5), мы определяем аналитическую функцию связи через представление (1) со спектральной плотностью, выраженной через $W$, что эквивалентно вычитанию вклада интеграла по "нефизическому разрезу,'

$$
\mathcal{A}^{(2)}(x)=-\frac{1}{b(1+\mathcal{W}(x))}-\frac{1}{\pi} \int_{0}^{1} \frac{\mathcal{R}(\sigma) d \sigma}{\sigma-x} .
$$

Заметим, что итеративное приближение (4) обладает несколько иной структурой (кроме полюса оно имеет логарифмическую точку ветвления при $x_{c}=e^{-b}$ ) призрачных сингулярностей. Тем не менее в работе [11] было установлено, что аналитизированное итеративное двухпетлевое приближение численно очень близко ${ }^{3)}$ к точному (6). С практической точки зрения это означает, что для $\mathcal{A}^{(2)}(x)$ можно использовать явное выражение (1) со спектральной плотностью

$$
\begin{aligned}
\rho_{\mathrm{P} \Gamma}^{(2)}(L) & =\frac{I(L)}{R^{2}(L)+I^{2}(L)} ; \quad L=\ln \frac{\sigma}{\Lambda^{2}}, \\
R(L) & =L+b \ln \sqrt{\left(1+\frac{L}{b}\right)^{2}+\left(\frac{\pi}{b}\right)^{2}}, \quad I(L)=\pi+b \arccos \frac{b+L}{\sqrt{(b+L)^{2}+\pi^{2}}} .
\end{aligned}
$$

В то же время в работе [12] было отмечено, что точное трехпетлевое решение (с преобразованной по Паде́ бета-функцией) также может быть выражено через функцию Ламберта:

$$
\begin{aligned}
a^{(3)}(x) & =-\frac{1}{b} \frac{1}{1-c+W(z)} ; \\
z & =-e^{-\ln x / b+c-1}, \quad c=\frac{\beta_{3} \beta_{1}}{\beta_{2}^{2}} .
\end{aligned}
$$

Ниже, обрашаясь к трехпетлевому случаю, будем подразумевать $\overline{\mathrm{MS}}$-схему с $\beta_{3}=$ $2857 / 2-5033 n_{f} / 18+325 n_{f}^{2} / 54$, здесь (при $\left.n_{f}=3\right) \beta_{3}=3863 / 6=643.833, c=1.415$.

\footnotetext{
3) $3 \div 4$-процентное отличие может быть скомпенсировано $8 \%$-й коррекцией значения $\Lambda$.
} 
2.3. Стабильность аналитической связи в ИК-области. Аналитическая связь $\mathcal{A}(x)$ обладает рядом важных свойств в ИК-области. Она оказывается довольно стабильной при $Q<\Lambda$ по отношению к вкладам от высших петель и относительно схемной зависимости. Предельное значение

$$
\mathcal{A}(0)=1
$$

оказьвается универсальным ${ }^{4)}$ в этом отношении.

Это замечательное свойство универсальности было впервые обнаружено в самой первой из наших работ [1] этого направления. Позднее более детальный анализ $[2,14,11,12]$ показал, что значение $\mathcal{A}(0)$ оказывается нечувствительным не только к высшим членам в бета-функции, но также и к деталям структуры призрачной сингулярности, устраняемой аналитизацией. А эта структура зависит от приближений.

Вместо корневой точки ветвления точного двухпетлевого решения (5), итеративное двухпетлевое решение (4) обладает полюсом при $x=1$ и логарифмическим ветвлением при $x_{*}=e^{-b}$. В трехпетлевом случае аппроксимированное по Паде́ решение имеет полюс и точку ветвления вместо сингулярности вида $(\ln x)^{-1 / 3}$ у решения с нетрансформированной бета-функцией.

Тем не менее во всех этих случаях окончательные аналитизированные результаты для $\mathcal{A}(x)$ обладают свойством $(9)$, а их ИК-поведения в интервале $(0,1)$ весьма близки [10] друг к другу. Процедура аналитизации, “сглаживающая все острые углы", уравнивает их всех.

Как видно, спектральность, ограничивая поведение инвариантной связи верхней границей, “держит ее в области достаточно малых значений”. Так, например, обычная бета-функция в $\overline{\mathrm{MS}}$-схеме при $n_{f}=3$

$$
\beta(a)=-a^{2}\left(1+0.790 a+0.883 a^{2}\right) \sim-\alpha^{2}\left(1+0.566 \alpha+0.453 \alpha^{2}\right)
$$

выглядит "вполне прилично": ее последовательные члены достаточно быстро убывают в достижимой на опыте физической области $\alpha \leqslant 0.4(a \leqslant 0.3)$. Здесь уместно ввести “бета-функцию для аналитической связи"

$$
\frac{d \mathcal{A}(x)}{d \ln x}=\beta_{\text {ан }}(\mathcal{A}(x)),
$$

являющуюся трансцендентной неаналитической функцией $\beta_{\text {ан }}(\mathcal{A})$ своего единственного аргумента с фиксированной точкой при $\mathcal{A}=1$. В однопетлевом случае эта функция может быть проанализирована достаточно просто (см. ниже формулы $(14)$ и (15)). При этом оказывается, что ее максимальное значение $\beta_{\text {ан }}(1 / 2)=1 / 12$ численно весьма близко (это свойство остается и в высших петлевых приближениях) к выражению (10), взятому при $a=0.3$.

Напомним, что связь между аналитичностью рассматриваемого типа в комплексной плоскости для физической амплитуды и ее ограниченностью обсуждалась ранее (см., например, работы [15], а также $[16, \S 11.3])$ в контексте анализа поведения низкоэнергетических характеристик рассеяния адронов.

\footnotetext{
4) Эффективное число ароматов $n_{f}$ в точке полюса равно 3 , что дает $\alpha_{\text {ан }}(0)=1.396$.
} 
2.4. Аналитизация наблюдаемых. В работах $[8,9]$ был предложен особый рецепт для аналитизации наблюдаемой $M(s)$.

Во-первых, следует выразить $M(s)$, возможно определенную во времениподобной области, через некоторую вспомогательную функцию $F\left(Q^{2}\right)$ пространственноподобного аргумента ${ }^{5)}$, обладаюшую свойствами аналитичности в $Q^{2}$-плоскости, совместными со спектральным представлением типа Челлена-Лемана. Эта функция после применения обычного ренормгруппового механизма приобретает форму разложения по степеням инвариантной связи:

$$
F(a(x))=\sum_{n} f_{n} a^{n}(x)
$$

Во-вторых, согласно предписанию причинная аналитизация функции $F(a)$ выполняется по правилу

$$
F(a(x)) \Rightarrow \mathcal{F}(x)=\sum_{n} f_{n} \mathcal{A}_{n}(x)
$$

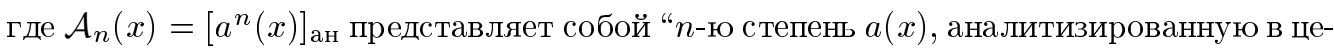
лом". З Десь каждая функция $\mathcal{A}_{n}(x)$ удовлетворяет представлению Челлена-Лемана со спектральной плотностью $\rho_{n}(\sigma)$, определенной как $\Im a^{n}(-\sigma)$.

Отметим, что разложение $(13)$ не является степенным, так как $\mathcal{A}_{n}(x) \neq[\mathcal{A}(x)]^{n}$ при $n \neq 1$. Рецепт $(12) \Rightarrow(13)$ меняет природу разложения!

Важной особенностью этого рецепта, приводящего к АТВ, является высокая стабильность его результатов относительно учета высших петлевых поправок $[8,9]$ и соответственно относительно схемной зависимости [10]. Мы собираемся показать, что природа этих физически важных свойств лежит в изменении вида разложения теории возмущений.

Нестепенная последовательность $\left\{\mathcal{A}_{n}(x)\right\}$ является асимптотической в УФ-области, так как

$$
\mathcal{A}_{n+1}(x) \simeq[\ln x]^{-(n+1)}=o\left(\mathcal{A}_{n}(x)\right), \quad x \rightarrow \infty
$$

Это означает, что ряд (13) может рассматриваться как асимптотическое разложение функции $\mathcal{F}(x)$ по асимптотической последовательности $\left\{\mathcal{A}_{n}(x)\right\}$. Свойства его сходимости определяются, с одной стороны, коэффициентами разложения $f_{n}$, вычисленными на основе $n$-петлевых диаграмм Фейнмана, а с другой стороны, свойствами последовательности $\left\{\mathcal{A}_{n}(x)\right\}$. Обратимся к рассмотрению последней.

\section{3. ПОСЛЕДОВАТЕЛЬНОСТЬ ФУНКЦИЙ РАЗЛОЖЕНИЯ}

3.1. Однопетлевые функции разложения. Наиболее простая последовательность состоит из аналитизированных степеней однопетлевой функции связи пКХД $a(x)$.

\footnotetext{
5) Так, например, отношение поперечных сечений для $e^{+} e^{-}$-аннигиляции $R(s)$ выражают через функцию Адлера $D\left(Q^{2}\right)$.
} 
Кроме аналитического инвариантного заряда $[a(x)]_{\text {ан }}=\mathcal{A}(x)($ см. $(2))$ эта последовательность содержит функции $\mathcal{A}_{n}(x)$ :

$$
\begin{aligned}
& a^{2}(x)=\frac{1}{\ln ^{2} x} \Rightarrow \mathcal{A}_{2}(x)=\frac{1}{\ln ^{2} x}-p_{2}(x) ; \quad p_{2}(x)=\frac{x}{(1-x)^{2}}=p_{2}\left(\frac{1}{x}\right) ; \\
& \mathcal{A}_{3}(x)=\frac{1}{\ln ^{3} x}+\frac{x}{(1-x)^{3}}-\frac{1}{2} \frac{x}{(1-x)^{2}} ; \quad \mathcal{A}_{4}(x)=\frac{1}{\ln ^{4} x}-p_{2}^{2}(x)-\frac{p_{2}(x)}{6} ; \ldots
\end{aligned}
$$

- “аналитизированные степени", обладаюшие симметрией $\mathcal{A}_{n}(x)=(-1)^{n} \mathcal{A}_{n}(1 / x)$ и связанные рекуррентным соотношением, содержашим оператор $\mathcal{D}=-x(d / d x)$,

$$
\mathcal{A}_{n+1}(x)=\frac{1}{n} \mathcal{D} \mathcal{A}_{n}(x)=\frac{1}{n !}\left(-x \frac{d}{d x}\right)^{n+1} \ln \mathcal{A}_{0}(x) ; \quad \mathcal{A}_{0}(x)=\frac{x-1}{x \ln x},
$$

где $\mathcal{A}_{0}$ играет роль производящей функции. В частности,

$$
\frac{d \mathcal{A}(x)}{d \ln x}=-\mathcal{A}_{2}(x) \equiv \beta_{\mathrm{aH}}^{(1)}(\mathcal{A}(x))=\beta_{\mathrm{aH}}^{(1)}(\mathcal{A}(1 / x)),
$$

здесь $\beta_{\mathrm{aн}}^{(1)}(\mathcal{A})$ не является аналитической функцией своего аргумента.

Попытка выразить $\mathcal{A}_{n}(x)$ через аналитическую функцию связи $\mathcal{A}(x)$ дает "смешанное" представление

$$
\begin{aligned}
& \mathcal{A}_{2}(x)=\mathcal{A}^{2}(x)-\frac{2}{1-x} \mathcal{A}(x)+\frac{1}{1-x} \\
& \mathcal{A}_{3}(x)=\mathcal{A}^{3}(x)-\frac{3}{1-x} \mathcal{A}^{2}(x)+\frac{3}{(1-x)^{2}} \mathcal{A}(x)-\frac{x+2}{2(1-x)^{2}}, \ldots
\end{aligned}
$$

сочетающее полиномиальную и непертурбативную (через аргумент $x=Q^{2} / \Lambda^{2}$ ) зависимости. Можно показать, что это представление не интересно с прагматической точки зрения (см. ниже п. 3.2).

В двухпетлевом случае, для того чтобы связать решение (6) с высшими аналитизированными степенями, следует формально воспользоваться оператором

$$
\mathcal{D}_{2}=\frac{1}{b} \frac{\partial}{\partial W}=-\frac{1+W}{W} \cdot x \frac{\partial}{\partial x},
$$

так что

$$
\mathcal{A}_{n+1}^{(2)}(x)=\frac{1}{n !} \mathcal{D}_{2}^{n} \mathcal{A}_{n}^{(2)}(x) .
$$

Заметим, что в уравнении (15) $\mathcal{D}$ можно рассматривать как оператор дифференцирования по “эффективному времени" $t=\ln x$. Подобная интерпретация в двухпетлевом случае

$$
\mathcal{D}_{2}=\frac{1}{1+b a^{(2)}\left(e^{t}\right)} \cdot \frac{d}{d t} \equiv \frac{d}{d \tau} ; \quad \tau=t+b \int_{0}^{t} a\left(e^{t^{\prime}}\right) d t^{\prime},
$$

приводит к новой переменной $\tau(t)$, выступаюшей в роли "эффективного двухпетлевого времени" $\tau=t_{2}$. При больших значениях $t$ имеем $\tau \simeq t+b \ln t$. Однако выражение для $\mathcal{D}_{2}$ требует уточнения при $0<x<1$. 
3.2. Вычитающие структуры и поведение при малых $Q$. Рациональные структуры $p_{n}(x)=\mathcal{A}_{n}(x)-a^{n}(x)$, которые вычитают призрачные сингулярности,

$$
\begin{aligned}
& p_{1}(x)=\frac{1}{1-x} ; \quad p_{2}(x)=\frac{x}{(1-x)^{2}} ; \quad p_{3}(x)=\frac{x(1+x)}{2(1-x)^{3}}=\frac{1+x}{1-x} \cdot \frac{p_{2}(x)}{2} ; \\
& p_{4}(x)=p_{2}^{2}(x)-\frac{p_{2}(x)}{6}=-\frac{x\left(x-x_{+}\right)\left(x-x_{-}\right)}{6(1-x)^{4}} \quad\left(x_{ \pm}=2 \pm \sqrt{3}\right) ; \ldots,
\end{aligned}
$$

связаны рекуррентным соотношением, аналогичным (15), и, за исключением $p_{1}$, обладают (анти)симметрией при замене $x \rightarrow 1 / x$.

Как следует из этого рекуррентного соотношения, все $p_{n \geqslant 2}(x)$ при $x=0$ имеют нуль первого порядка, что обеспечивает выполнение важного свойства

$$
\mathcal{A}_{n}(0)=0 ; \quad n \geqslant 2
$$

которое также имеет место в двух- и трехпетлевом случаях.

Для количественной оценки рассмотрим поведение $\mathcal{A}_{n}(x)$ при $x=1+\epsilon ; \epsilon \ll 1$. В однопетлевом случае с помощью выражений (20) это может быть сделано явно:

$$
\mathcal{A}(x) \simeq \frac{1}{2}-\frac{\epsilon}{12} ; \quad \mathcal{A}_{2}(x) \simeq \frac{1}{12}+\frac{19}{60} \epsilon^{2} ; \quad \mathcal{A}_{3}(x) \simeq+\frac{\epsilon}{240} ; \quad \mathcal{A}_{4}(x) \simeq-\frac{1}{720}+\frac{\epsilon^{2}}{3042}
$$

Эти численные результаты оказываются довольно полезными. Вместе с условием (21) они показывают, что в “области малых $Q$ ” (т.е. при $Q \leqslant \Lambda$ ) мы имеем важное свойство

$$
\mathcal{A}_{n}(x) \ll \mathcal{A}^{n}(x)
$$

которое в конечном итоге ответственно за низкий уровень чувствительности результатов АТВ по отношению к высшим петлевым поправкам и схемным эффектам (см., например, заключения в работах [8-10]). Последнее соотношение оказывается справедливым и при учете высших петель.

Прибегать к выражениям вида (5), (8) и (18) для явного анализа последовательностей $\left\{\mathcal{A}_{n}(x)\right\}$ в двух- и трехпетлевом случаях не целесообразно. Для ориентировки мы использовали численные вычисления через соответствующий спектральный интеграл. Их результаты показывают, что в интервале $Q<\Lambda$ в дополнение к соотношению (22) имеет место оценка

$$
\mathcal{A}_{k+1}(x) \cong[\mathcal{A}(x)]^{1+2 k}
$$

Другими словами, в “области малых $Q$ ” реальный параметр разложения численно ближе к $\mathcal{A}^{2}(x)$, чем к $\mathcal{A}(x)^{6)}$. В то же время с ростом $Q / \Lambda$ и при уменьшении степенных слагаемых $\mathcal{A}_{n}(x)$ стремятся к своим естественным пределам $[\mathcal{A}(x)]^{n} \simeq a^{n}(x)$.

\footnotetext{
6) Отсюда следует, что "смешанное" представление $\mathcal{A}_{n}$ по степеням функции $\mathcal{A}$ с непертурбативными коэффициентами неприемлемо из-за больших численных сокрашений в правой части соотношений, аналогичных уравнению (17).
} 
Более того, как это следует из представления (20) и свойства сингулярности $\ln ^{-n} x$ при $x=0$, функции $\mathcal{A}_{n+2}(x)$ имеют ровно $n$ нулей в интервале $(0, X(\Lambda))$. Здесь $X(\Lambda)$ является верхней границей области, где степенные непертурбативные поправки порядка $x^{-1}=\Lambda^{2} / Q^{2}$ еше сушественны. Следовательно, обсуждаемая последовательность состоит из квазиосциллирующих функций. Это обстоятельство делает задачу оценки остаточного члена (погрешности) в асимптотическом разложении (13) более сложной, что характерно для асимптотического разложения в смысле Эрдейи.

Заметим еше, что последовательности $\left\{\mathcal{A}_{n}(x)\right\}$ в рассмотренных случаях обладают особым свойством. Их последуюшие члены связаны дифференциальными соотношениями (15) и (18).

\section{4. ОБСУЖДЕНИЕ}

Мы проанализировали частную версию "причинной, $Q^{2}$-аналитичной теории возмушений" - АТВ-версию, в которой по соглашению, впервые введенному в работе [8], для аналитизации наблюдаемых используется асимптотическая последовательность $\left\{\mathcal{A}_{n}(x)\right\}$. Ее можно рассматривать как "нестепенную аналитизацию", в отличие от другой - "степенной", осушествляемой с помошью последовательности $\left\{\mathcal{A}^{n}(x)\right\}$, т.е. с помошю замены

$$
F(a(x)) \Rightarrow F(\mathcal{A}(x))=\sum_{n} f_{n}[\mathcal{A}(x)]^{n} .
$$

Именно нестепенная аналитизация приводит к петлевой и схемной стабильности. Между тем степенная аналитизация (23) дает заметные отклонения от пКХД лишш в ИК-области.

С теоретической точки зрения эта последняя, технически более простая версия имеет преимушество в отношении согласованности аналитизации со структурой ренормгруппь (см. ниже п. 4.2). Для пояснения этого утверждения обратимся к структуре алгоритма РГ-метода и укажем на возможность "некоммутативности" аналитизации с некоторыми его элементами.

4.1. О произволе процедуры аналитизации. Процедура ренормгруппового метода состоит из нескольких этапов:

I. Вычисление бета-функции(й) и аномальных размерностей.

II. Решение РГ-дифференциальных уравнений (РГДУ) для инвариантной связи $a(x)$.

III. Решение РГДУ для других функций $f\left(Q^{2}, a\right)$, например амплитуд пропагаторов, эффективных масс и "наблюдаемых" 7$)$, с использованием явных выражений для $\beta$-функции, найденной на этапе I, или инвариантной связи $a(x)$, полученной на этапе II. В результате вместо $f$ возникает функция $F(a(x))$, представленная в виде степенного ряда (начинаюшегося, возможно, с логарифмического члена).

Аналитизацию инвариантной связи нужно рассматривать как дополнительный этап, следуюший за II,

IIa: $a(x) \Rightarrow \mathcal{A}(x)$.

\footnotetext{
7) Таких как функции Адлера, моменты структурных функций и т.д.
} 
Однако аналитизация пропагаторов и наблюдаемых может быть теперь выполнена различными способами: либо модификацией этапа III -

$\mathrm{III}_{\mathrm{M}}$. Использование выражения $\mathcal{A}(x)$ при решении РГДУ для функций $f\left(Q^{2}, a\right)$; либо как дополнительный этап, например,

IV $\mathrm{ATB}$. Аналитизирование результата этапа III, т.е. применение процедуры аналитизации к степенному разложению для $F(a(x))$.

Последовательность

$$
\mathrm{I}+\mathrm{II}+\mathrm{IIa}+\mathrm{III}+\mathrm{IV}_{\mathrm{ATB}}=\mathrm{ATB}
$$

была использована в работах [8-10]. Именно эта процедура привела к нестепенному асимптотическому разложению (13).

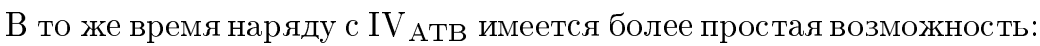

$\mathrm{IV}_{\text {ан }}$. Подстановка явного выражения $\mathcal{A}(x)$ (как в $\left.(23)\right)$ в результат этапа III.

Таким образом, набор

$$
\mathrm{I}+\mathrm{II}+\mathrm{IIa}+\mathrm{III}+\mathrm{IV}_{\mathrm{aH}}=\mathrm{A} \text { ИC }
$$

(АИС означает "аналитизацию инвариантной связи”) также может быть использован для аналитизации наблюдаемых. Такая процедура приводит к асимптотическому разложению (23), отличающемуся от обычного (12) только заменой $a(x) \Rightarrow \mathcal{A}(x)$.

Поэтому причинная аналитизация наблюдаемых оказывается неоднозначной процедурой. Примечательно, что отмеченный произвол имеет функциональную природу и не сводится к возможности введения какого-либо подгоночного параметра.

4.2. Аналитизация и структура РГ . Набор I + II + IIa $+\mathrm{III}_{\mathrm{M}}$ содержит внутреннее противоречие. Так, операция $\mathrm{III}_{\mathrm{M}}$, примененная, например, к амплитуде глюонного пропагатора ${ }^{8)}$, приводит к выражению, которое оказывается несовместным с результатом этапа ІІа. На однопетлевом уровне III $_{\mathrm{M}}$ дает $[14]$

$$
d_{\mathrm{P} \Gamma}^{i}(x)=[a(x)]^{\nu_{i}} \Rightarrow\left[\mathcal{A}_{0}(x)\right]^{\nu_{i}},
$$

где $\mathcal{A}_{0}(x)$ определено в (15). Однако, как это следует из основных РГ-соотношений, произведение вершинной функции и соответствуюших степеней пропагаторов образует инвариантный заряд. В применении к нашему случаю такое произведение даст $\mathcal{A}_{0}(x)$, а не аналитический инвариантньй заряд $a_{\text {ан }}(x)=\mathcal{A}(x)$, использованный как исходный.

Аналогично этому возникает тонкость при выполнении этапа IV Атв. Дело в том, что для некоторых объектов (например, для амплитуды пропагатора) результат этапа III на однопетлевом уровне начинается с дробной степени (или логарифма) $a(x)$, что дает нефизическую особенность в виде точки ветвления. Аналитизация таких выражений как $[\ln x]^{-\nu}$ эквивалентна вычитанию вклада разреза, т.е. приводит к специфической двухчленной структуре ${ }^{9)}$. Легко видеть, что надлежащее произведение таких структур не может дать выражение (2) для инвариантного заряда. Это наблюдение, трактуемое буквально, означает, что процедура АТВ также вступает в противоречие со структурой РГ.

\footnotetext{
8) Сp. с приемом работы [11], эквивалентным этапу $\mathrm{IV}_{\text {ан }}$.

9) За точными выражениями мы отсылаем, например, к работам $[6,11]$.
} 


\section{5. ЗАКЛЮЧЕНИЕ}

1. Наш анализ в разделе 3 показал, что разложение (13) “версии АТВ" для наблюдаемой в общем случае является асимптотическим разложением по нестепенной последовательности $\left\{\mathcal{A}_{n}(x)\right\}$. Последняя может иметь довольно разные свойства в различных областях переменной $x$. В УФ-области она близка к степенной последовательности $\left\{a^{n}(x)\right\}$, обычно используемой в современных вычислениях КТП по теории возмушений. Следовательно, сходимость в УФ-области полностью определяется коэффициентами разложения $f_{n}$. С другой стороны, в ИК-области последовательность $\left\{\mathcal{A}_{n}(x)\right\}$ имеет более сложную структуру. При $0<x<1$ поведение функций $\mathcal{A}_{n}(x), n \geqslant 3$, является осциллируюшим. Благодаря этому вклад высших членов в АТВ-разложении подавлен. Разложение (13) в ИК-области, по всей видимости, подобно асимптотическому разложению в смысле Эрдейи. Этот предварительный вывод дает надежду на возможность использования пертурбативного подхода в КХД в области $Q \sim 1$ ГэВ, где эффективная функция связи КХД не является малой величиной.

2. В п. 4.1 мы показали, что общая программа инвариантной аналитизации, будучи полностью определенной процедурой для эффективного заряда, не является достаточно "жесткой”, когда она применяется к другим объектам. В частности, она содержит произвол при аналитизации наблюдаемых.

Этот произвол и “близость к противоречию”, обсуждавшаяся в п. 4.2, указывают на актуальность поиска дополнительного ограничения во всем причинном аналитическом подходе. В то же время возможность, предоставляемая АТВ, слишком интересна, чтобы "отказаться от нее без борьбы".

3. По нашему мнению, еще одним курьезным уроком рассмотренной нестепенной конструкции является полуколичественное наблюдение того, что АТВ-подход эквивалентен обычной пКХД-технике с довольно странной поправкой: “Ограничься в вьгислениях только лидирующим вкладом КХД," кстати, забывая при этом обо всех “головных болях", связанных с вычислением диаграмм высших порядков, со схемной зависимостью и сходимостью разложений. Эта интригуюшая возможность могла бы быть сформулирована как предположение, что пертурбативная КХД является эффективной теорией, а вклады высших порядков не имеют ясного физического содержания ${ }^{10)}$.

Благодарности. Автору приятно поблагодарить И. Л. Соловцова за важные советы и помощь в численных расчетах, а также И. Я. Арефьеву, Б. А. Арбузова, В. С. Владимирова, Д. И. Казакова, А. Л. Катаева, Н. В. Красникова, Б. А. Маградзе, А. В. Нестеренко и А.А. Славнова за интерес к работе и полезное обсуждение.

Работа выполнена при частичной поддержке РФФИ (гранты №96-01-01860, №96-15-96030) и INTAS (грант №96-0842).

\footnotetext{
10) Подобно, например, вкладам высших порядков теории возмущений для эфффективного четырехфермионного лагранжиана Ферми в КТП и для модельного гамильтониана БКШ в теории сверхпроводимости.
}

3 Теоретическая и математическая физика, т. 119, № 1, 1999 г. 


\section{Список литературы}

[1] D. V. Shirkov, I. L. Solovtsov. Краткие сообщения ОИЯИ. 1996. № 2[76]. С. 5; hep-ph/9604363.

[2] D. V. Shirkov, I. L. Solovtsov. Phys. Rev. Lett. 1997. V. 79. P. 1209; hep-ph/9704333.

[3] G. Källen. Helv. Phys. Acta. 1952. V. 25. P. 417.

[4] H. Lehmann. Nuovo Cim. 1954. V. 11. P. 342.

[5] Н. Н. Боголюбов, Д. В. Ширков. Введение в теорию квантованных полей. М.: Наука, 1984.

[6] Н.Н. Боголюбов, А. А. Логунов, Д. В. Ширков. ЖЭТФ. 1959. Т. 37. С. 805.

[7] D. V. Shirkov. Lett. Mat. Phys. 1976. V. 1. P. 179; Lett. Nuovo Cim. 1977. V. 18. P. 452.

[8] K. A. Milton, I. L. Solovtsov, O.P. Solovtsova. Phys. Lett. B. 1977. V. 415. P. 104; hep-ph/9706409.

[9] K. A. Milton, I. L. Solovtsov, O.P. Solovtsova. Phys. Lett. B. 1998. V. 439. P. 421; hep-ph/9809510; The Gross-Llewellyn Smith sum rule in the analytic approach to perturbative QCD. Preprint OKHEP-98-07, Oklahoma Univ.; hep-ph/9809513.

[10] I. L. Solovtsov, D. V. Shirkov. Phys. Lett. B. 1998. V. 442. P. 344; hep-ph/9711251.

[11] B. A. Magradze. The gluon propagator in analytic perturbation theory. Доклад на конференции "Кварки-98", Суздаль, Май 1998; hep-ph/9808247.

[12] E. Gardi, G. Grunberg, M. Karliner. Can the QCD running coupling have a causal analyticity structure? hep-ph/9806462.

[13] R. M. Corless et al. Adv. Comput. Math. 1996. V. 5. P. 329.

[14] D. V. Shirkov. Nucl. Phys. B (Proc. Suppl.). 1998. V. 64. P. 106; hep-ph/9708480.

[15] А. В. Ефремов, Д. В. Ширков, Чжу Хун-юань. ЖЭТФ. 1961. Т. 41. С. 603; A. V. Efremov, D. V. Shirkov, H. Y. Tzu. The pion-pion scattering at low energy. Preprint D-757. Dubna: JINR, 1960; Scientia Sinica. 1961. V. 10. P. 812.

[16] Д. В. Ширков, В. А. Мещеряков, В. В. Серебряков. Дисперсионные теории сильных взаимодействий при низких энергиях. М.: Наука, 1967.

[17] A. В. Нестеренко. Моменты структурных функций в аналитическом подходе в квантовой хромодинамике. Дипломная работа. Физ. фак. МГУ, 1998.

Поступила в редакцию 14.X.1998 г. 